\title{
Surface gravity of neutron stars and strange stars
}

\author{
M. Bejger ${ }^{1}$ and P. Haensel ${ }^{1,2}$ \\ ${ }^{1}$ N. Copernicus Astronomical Center, Polish Academy of Sciences, Bartycka 18, 00-716 Warszawa, Poland \\ 2 LUTH du CNRS, Observatoire de Paris, 92195 Meudon Cedex, France \\ e-mail: haensel@camk.edu.pl
}

Received 20 October 2003 / Accepted 28 February 2004

\begin{abstract}
The upper bound on the value of the surface gravity, $g_{\mathrm{s}}$, for neutron stars with equations of state respecting $v_{\text {sound }} \leq c$, is derived. This bound is inversely proportional to the maximum allowable mass $M_{\max }$, and it reads $g_{\mathrm{s}} \leq$ $1.411 \times 10^{15}\left(M_{\max } / M_{\odot}\right)^{-1} \mathrm{~cm} \mathrm{~s}^{-2}$. It implies an absolute upper bound $7.4 \times 10^{14} \mathrm{~cm} \mathrm{~s}^{-2}$ if one uses the $2 \sigma$ lower bound on the neutron mass measured recently in 4U1700-37, 1.9 $M_{\odot}$. A correlation between $g_{\mathrm{s}}$ and the compactness parameter $2 G M / R c^{2}$ for baryonic stars is analyzed. The properties of $g_{\mathrm{s}}$ of strange quark stars and its upper bounds are discussed using the scaling properties of the strange-star models.
\end{abstract}

Key words. dense matter - equation of state - stars: neutron

\section{Introduction}

Gravitational acceleration on the stellar surface, usually called the surface gravity, is an important parameter of the theory of stellar atmospheres, in particular the atmospheres of neutron stars (see, e.g., Zavlin \& Pavlov 2002). For neutron stars, $g_{\mathrm{s}}$ should be defined taking into account the space-time curvature. The value of $g_{\mathrm{s}} \sim$ few $\times 10^{14} \mathrm{~cm} \mathrm{~s}^{-2}$ is involved the relation between internal and surface temperature of neutron stars (Potekhin et al. 2003 and references therein).

The surface gravity of neutron stars is by many orders of magnitude larger than for other stars; it is $\sim 10^{5}$ times larger than for white dwarfs, and $10^{8}$ times stronger than the gravity at the solar surface. As we show in the present paper, at a given neutron-star mass the value of $g_{\mathrm{s}}$ depends very strongly on the largely unknown equation of state (EOS) of dense matter at supra-nuclear densities. It is therefore of interest to derive upper bounds on $g_{\mathrm{s}}$. In Sect. 2 we derive such upper bounds on $g_{\mathrm{s}}$ resulting from the condition of subluminality of the EOS (speed of sound not exceeding $c$ ). We show how an upper bound on $g_{\mathrm{s}}$ can be obtained from measured neutron star masses. In Sect. 3 we analyze the EOS-dependence of $g_{\mathrm{s}}$ for a set of 31 models of dense matter and compare the maximal surface gravity, reached at the maximum allowable mass, with subluminal upper bounds. In Sect. 4 we consider surface gravity of strange quark stars. We derive a scaling formula for $g_{\mathrm{s}}$ of strange stars and use it to relate the maximum surface gravity for various models of such stars. Concluding remarks are presented in Sect. 5.

Send offprint requests to: $\mathrm{M}$. Bejger, e-mail: bejger@camk.edu.pl

\section{Bounds on surface gravity}

It is well known that (see, e.g., Shapiro \& Teukolsky 1983)

$g_{\mathrm{s}}=\frac{G M}{R^{2} \sqrt{1-2 G M / R c^{2}}}$

where $M$ is the gravitational mass of the star and $R$ is the stellar circumferential radius, and effects of rotation are neglected. For a "standard neutron star" with $M=1.4 M_{\odot}$ and $R=10 \mathrm{~km}$ one has $g_{\mathrm{s}}=2.43 \times 10^{14} \mathrm{~cm} \mathrm{~s}^{-2}$. It is therefore convenient to measure $g_{\mathrm{s}}$ in units of $10^{14} \mathrm{~cm} \mathrm{~s}^{-2}$, and to use $g_{\mathrm{s}, 14} \equiv g_{\mathrm{s}} /\left(10^{14} \mathrm{~cm} \mathrm{~s}^{-2}\right)$.

Let us introduce the dimensionless compactness parameter $x \equiv r_{\mathrm{g}} / R=2 G M / R c^{2}$ where $r_{\mathrm{g}}$ is the Schwarzschild radius. Then

$g_{\mathrm{s}, 14}(x, M)=15.21 \frac{x^{2}}{\sqrt{1-x}} \frac{M_{\odot}}{M}$

Generally, physical theories have to be causal. In the literature, the condition of causality is usually replaced by the constraint of subluminality of the EOS, $v_{\text {sound }}=(\mathrm{d} P / \mathrm{d} \rho)^{1 / 2} \leq c$ (see, e.g., Rhoades \& Ruffini 1974; Hartle 1978; Kalogera \& Baym 1996; Keiser \& Polyzou 1996; Glendenning 1997; Koranda et al. 1997; Haensel et al. 1999), but strictly speaking, subluminality is not equivalent to causality. For instance, Bludman \& Ruderman (1968) constructed causal and Lorenzinvariant EOSs with $v_{\text {sound }}>c$. In practice, the difference seems to be unimportant; the counterexamples of Bludman \& Ruderman (1968) correspond actually to a highly excited medium (Bludman \& Ruderman 1970). Nevertheless, for the sake of rigor, we will call an EOS respecting the condition 
$v_{\text {sound }} \leq c$ "subluminal", and an EOS violating this condition will be called "superluminal".

For subluminal EOSs of dense matter, a strict upper bound on $x$ is $x_{\max }=0.708$ (see, e.g., Haensel et al. 1999, and references therein). Using this value we get, from Eq. (2), an upper bound for a surface gravity of non-rotating neutron star of mass $M$,

$v_{\text {sound }} \leq c \quad \Longrightarrow \quad g_{\mathrm{s}, 14} \leq g_{\max , 14}^{\mathrm{SL}}=14.11 \frac{M_{\odot}}{M}$.

The maximal mass $M_{\max }$ obtained from the realistic EOS should be higher than the largest measured neutron star mass, $M_{\mathrm{obs}}^{\max }$. Known rotation frequencies of neutron stars are sufficiently small compared to the mass-shedding (Keplerian) limit, therefore we can use the non-rotating approximation for observed neutron stars. We thus have, in terms of $g_{\mathrm{s}}$, an upper bound based on the observations and subluminality conditions

$g_{\mathrm{s}, 14}<14.11 \frac{M_{\odot}}{M_{\mathrm{obs}}^{\max }}$.

The higher $M_{\mathrm{obs}}^{\max }$, the stronger the constraints on the maximum allowable $g_{\mathrm{s}}$ (the smaller the value of the upper bound). A precisely measured mass of the Hulse-Taylor pulsar, $(1.4408 \pm$ $0.0006) M_{\odot}$ (Weisberg \& Taylor 2003), gives the upper bound $9.79 \times 10^{14} \mathrm{~cm} \mathrm{~s}^{-2}$. According to Clark et al. (2002), neutron star in the high-mass X-ray binary $4 \mathrm{U} 1700-37$ has, at the $2 \sigma$ confidence level, $M_{\mathrm{obs}}>1.9 M_{\odot}$. This implies an upper bound lower than $7.4 \times 10^{14} \mathrm{~cm} \mathrm{~s}^{-2}$. It should be mentioned, however, that the result of Clark et al. (2002) has to used with care, because of large errors. Moreover, because the compact object in 4U1700-37 is neither an X-ray pulsar nor an X-ray burster, it could in principle be a low-mass black hole.

The expression on the right-hand-side of (4) deserves an additional comment. Apart from the numerical constant, it is identical to the upper bound on the frequency of stable rigid rotation of neutron stars with subluminal EOS (Koranda et al. 1997). An alternative derivation of an approximate (but very precise) upper bound on rotation frequency, relating it to the maximum stellar compactness (surface redshift) for subluminal EOS, was given by Haensel et al. (1999). In the latter work, the starting point was the "empirical formula" for the maximum rotation frequency. Its upper bound was also obtained by maximizing a function of $x=r_{\mathrm{g}} / R$.

\section{Equation of state of dense matter and surface gravity}

We have calculated $g_{\mathrm{s}}$ for neutron-star models based on a set of 31 baryonic EOSs of dense matter. The EOSs were obtained under different assumptions regarding the composition of the matter at $\rho \gtrsim 2 \rho_{0}$, where $\rho_{0}=2.7 \times 10^{14} \mathrm{~g} \mathrm{~cm}^{-3}$ is the normal nuclear density.

The EOSs can be subdivided into several groups. Within one group of models, the matter consists of nucleons and leptons (Baldo et al. 1997; Bombaci 1995; Balberg \& Gal 1997; Balberg et al. 1999; model I of Bethe \& Johnson 1974 (usually called BJI); Pandharipande 1971; Pandharipande \& Ravenhall 1989; Douchin \& Haensel 2001; Wiringa et al. 1988;

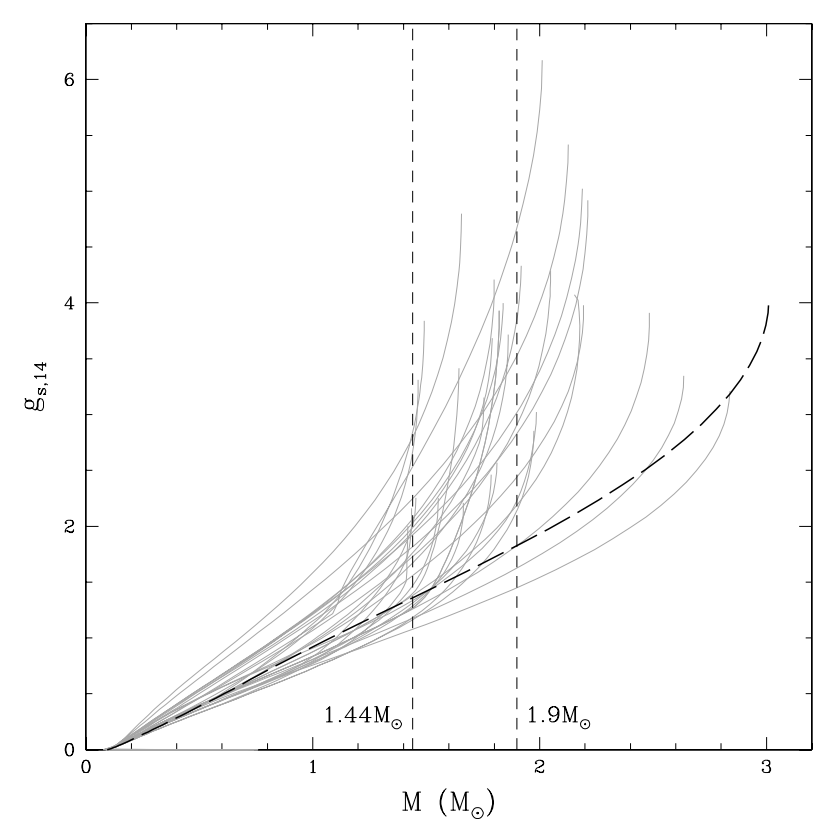

Fig. 1. Surface gravity in the units of $10^{14} \mathrm{~cm} \mathrm{~s}^{-2}$ against neutron-star mass for 31 EOSs of dense baryonic matter. Only stable configurations are shown, so that the curves terminate at the maximum allowable mass. Thick dashed line: maximally-stiff-core EOS. Remaining EOSs: thin lines. For further explanation see the text.

Walecka 1974; Haensel et al. 1980; Akmal et al. 1998). Within the second group, the matter is assumed to consist of nucleons, hyperons and leptons (Glendenning 1985; Balberg \& Gal 1997; Weber et al. 1991). The third group involves an exotic highdensity phase: de-confined quark matter mixed with baryonic matter (Glendenning 1997), pion condensate (Muto \& Tatsumi 1990 ) and kaon condensate (Kubis 2001). Finally, one EOS is the so called "maximally-stiff-core" (MSC) EOS. It consists of the BJI EOS below the baryon density $0.3 \mathrm{fm}^{-3}$, matched continuously to the EOS with $v_{\text {sound }}=c$ at higher density.

In Fig. 1 we show the surface gravity versus stellar (gravitational) mass for all 31 baryonic EOSs. The values of $g_{\mathrm{s}}$ for a given mass strongly depend on the EOS. For $M=1.44 M_{\odot}$, $g_{\mathrm{s}, 14}$ ranges from 1.1 to 2.8 , while for $M=1.9 M_{\odot}$ the predicted values are between 1.4 and 4.7.

We considered also three EOSs of self-bound absolutely stable strange quark matter, forming hypothetical strange stars (Zdunik et al. 2000; Dey et al. 1998) - they will be studied separately in Sect. 4.

Contrary to the significant scatter of the $g_{\mathrm{s}}(M)$ plots for baryonic stars, $g_{\mathrm{s}}(x)$ is much less EOS-dependent: it can be rather well (within better than $20 \%$ ) reproduced by the approximate formula

$g_{\mathrm{s}, 14} \simeq \frac{5 x^{5 / 4}}{\sqrt{1-x}}$.

Actually, the precision of this formula is much higher if we exclude the superluminal EOSs and the unrealistically stiff ones (too high incompressibility of nuclear matter at saturation) which yield $M_{\max } \gtrsim 2.5 M_{\odot}$. Putting then the upper bound $x_{\max }=0.708$ into (5) yields an approximate "realistic upper bound" of $6 \times 10^{14} \mathrm{~cm} \mathrm{~s}^{-2}$ on $g_{\mathrm{s}}$ of neutron stars. 
The maximum value of $g_{\mathrm{s}}$ for stable stars with a given EOS is reached at the maximum allowable mass. The values of $g_{\mathrm{s}, \max }$ for the selected EOSs are shown in Fig. 3. For comparison, we show also the absolute upper bounds, Eq. (3), at $M_{\max }$. The dense matter models were divided into groups denoted by specific symbols. Subluminal models involving only nucleons (o) give $g_{\mathrm{s}, \max } \simeq(3-5) \times 10^{14} \mathrm{~cm} \mathrm{~s}^{-2}$. The stiffer the EOS, the closer $g_{\mathrm{s}, \max }$ to the subluminal upper bound $g_{\max }^{\mathrm{SL}}\left(M_{\max }\right)$.

Subluminal hyperonic EOSs $(\bullet)$ give $g_{\mathrm{s}, \max }$ which is typically lower than $4 \times 10^{14} \mathrm{~cm} \mathrm{~s}^{-2}$. For these EOSs, $g_{\mathrm{s} \text {, max }}$ can be as small as one-fifth of the upper bound $g_{\max }^{\mathrm{SL}}\left(M_{\max }\right)$.

The subluminal EOSs with an exotic high-density phase $(\oplus)$ have relatively low $g_{\mathrm{s}, \max }$. A phase transition softens the EOS, lowering the radius. Simultaneously, however, the softening leads to a decrease of $M_{\max }$. The latter effect dominates over the former one. This strongly pushes up the upper bound $g_{\mathrm{s}}\left(M_{\max }\right)$. If the stellar interior consists mostly of a mixed quark-baryon phase (EOSs G6, G7, G8 in Fig. 3), then $g_{\mathrm{s}, \max } \simeq$ $2 \times 10^{14} \mathrm{~cm} \mathrm{~s}^{-2}$, only one fifth of $g_{\max }^{\mathrm{SL}}\left(M_{\max }\right)$.

The MSC EOS $(\odot)$ yields $g_{\mathrm{s}, \max }$ which is quite close to $g_{\mathrm{s}, \max }^{\mathrm{SL}}$. A similar situation occurs for the EOSs that give $M_{\max }$ models with superluminal cores, labeled $\star$. Their values of $g_{\mathrm{s} \text {, max }}$ range from $4 \times 10^{14} \mathrm{~cm} \mathrm{~s}^{-2}$ to $6 \times 10^{14} \mathrm{~cm} \mathrm{~s}^{-2}$. Note that even for those EOSs $g_{\mathrm{s}, \max }<g_{\mathrm{s}, \max }^{\mathrm{SL}}$. This is because the compactness parameter is always smaller than the upper bound for subluminal EOSs, $x\left(M_{\max }\right)<x_{\max }=0.708$, as is shown in Fig. 2.

\section{Surface gravity of strange stars}

The $g_{\mathrm{s}}(M)$ dependence for strange stars, presented in the upper panel of Fig. 4, is very different from that of baryonic stars, due to a different $R(M)$ dependence. The model dependence of $g_{\mathrm{s}}(M)$ for strange stars is very strong: at $1.44 M_{\odot}$, the value of $g_{\mathrm{s}, 14}$ ranges from 2.0 to 5.5. However, this strong EOS dependence can be explained in terms of the scaling properties of strange star models.

EOSs of self-bound quark matter (strange matter) are derived from various different models of the quark structure of hadrons. Despite differences between the underlying models, the EOSs relevant for stable models of strange stars can be well represented (fitted) by a linear relation between the pressure $P$ and the mass density $\rho$ (Zdunik 2000),

$P=a c^{2}\left(\rho-\rho_{\mathrm{s}}\right)$.

The parameters of stellar configuration, calculated for an EOS given by Eq. (6), are connected to those obtained for an EOS with $\rho_{\mathrm{s}}^{\prime} \neq \rho_{\mathrm{s}}$ by the scaling relations (Witten 1984; Haensel et al. 1986; Zdunik 2000). For example, points on the $M(R)$ curves are related by

$M=\left(\frac{\rho_{\mathrm{s}}^{\prime}}{\rho_{\mathrm{s}}}\right)^{\frac{1}{2}} M^{\prime}, \quad R=\left(\frac{\rho_{\mathrm{s}}^{\prime}}{\rho_{\mathrm{s}}}\right)^{\frac{1}{2}} R^{\prime}$.

Therefore, at a fixed $a$, the ratio $M / R$ (and therefore $x=r_{\mathrm{g}} / R$ ) does not depend on $\rho_{\mathrm{s}}$, and the maximum surface gravity of strange stars scales as

$g_{\mathrm{s}, \max }^{\prime}=\left(\frac{\rho_{\mathrm{s}}^{\prime}}{\rho_{\mathrm{s}}}\right)^{\frac{1}{2}} g_{\mathrm{s}, \max }$.

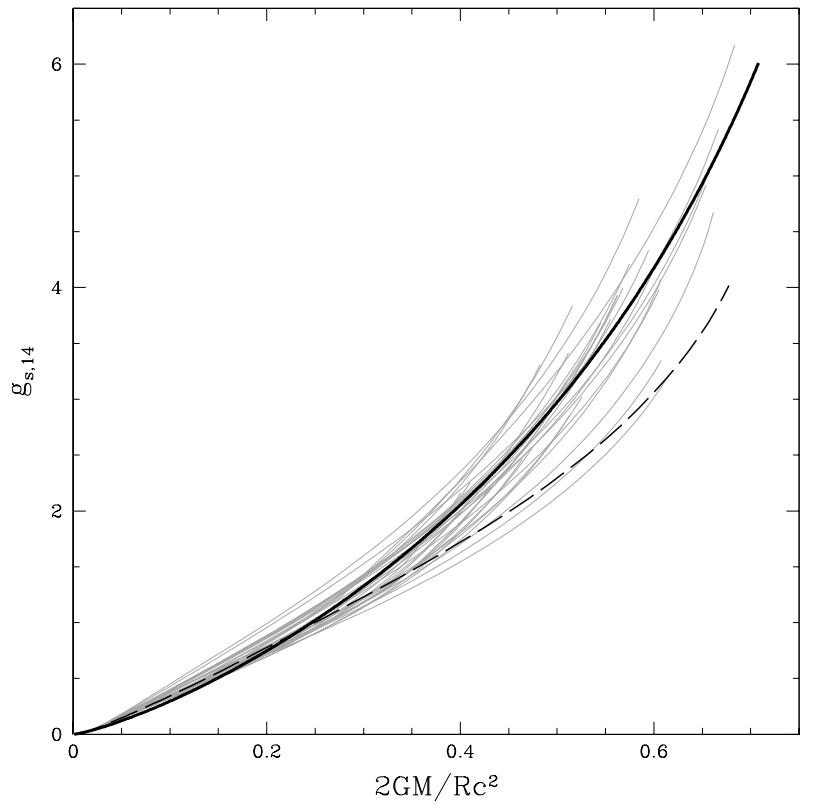

Fig. 2. Plots of $g_{\mathrm{s}, 14}$ versus compactness parameter $2 G M / R c^{2}$. Notation as in Fig. 1. Thick solid line represents approximate formula, Eq. (5).

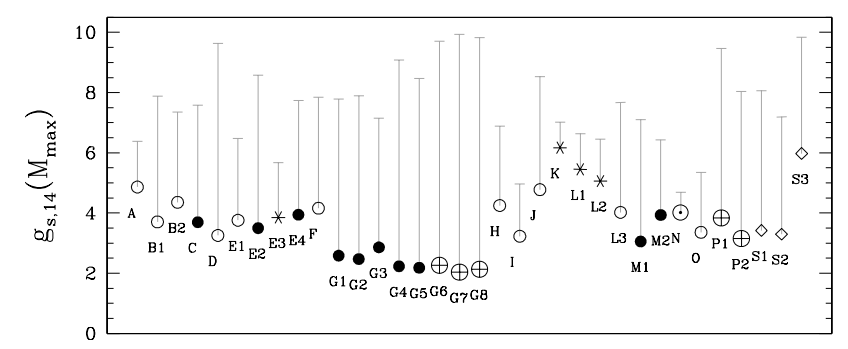

Fig. 3. Maximum values of surface gravity in units of $10^{14} \mathrm{~cm} \mathrm{~s}^{-2}$ (centers of the symbols at the bottom end of the vertical segments) for 34 baryonic and quark EOSs of dense matter, and the upper-bounds at the maximum allowable mass for these EOSs obtained from Eq. (3) (dashes at the upper end of the vertical segments). Nucleonic EOS: B1, B2 - Baldo et al. (1997); D - Bombaci (1995); E1, E3 Balberg \& Gal (1997), Balberg et al. (1999); C - model I of Bethe \& Johnson (1974); J - Pandharipande (1971); F - Pandharipande \& Ravenhall (1989); H Douchin \& Haensel (2001); L1, L2, L3 - Wiringa et al. (1988); 0 Walecka (1974); I - Haensel et al. (1980); A - Akmal et al. (1998). Hyperonic EOSs: G1-G5 - Glendenning (1985); E2, E4 - Balberg \& Gal (1997), Balberg et al. (1999); M1, M2 - Weber et al. (1991). EOSs with exotic high-density phase: G6-G8 - Glendenning (1997); K Kubis (2001); P1, P2 - Muto \& Tatsumi (1990). Strange quark matter: S1, S2 - Zdunik et al. (2000); S3 - Dey et al. (1998). N labels the MSC (maximally-stiff-core) EOS. For further explanations see the text.

The values of $a$ for the EOSs S1, S2, and S3 range within $0.30 \leq a \leq 0.46$. We have the exact ratio $g_{\mathrm{s}, \max }(\mathrm{S} 3) / g_{\mathrm{s}, \max }(\mathrm{S} 2)=1.81$, while the scaling factor $\left[\rho_{\mathrm{s}}(\mathrm{S} 3) / \rho_{\mathrm{s}}(\mathrm{S} 2)\right]^{1 / 2}=1.63$. The large difference in maximum surface gravities can therefore be accounted for by scaling with respect to $\rho_{\mathrm{s}}$; in this context, the $a$ dependence is sufficiently weak to be neglected in the simplest approach. As we see in the lower panel in Fig. 4, the precision of this scaling increases with decreasing strange star compactness. The scaling becomes very precise for $x<0.2$. The explanation of this behaviour is 

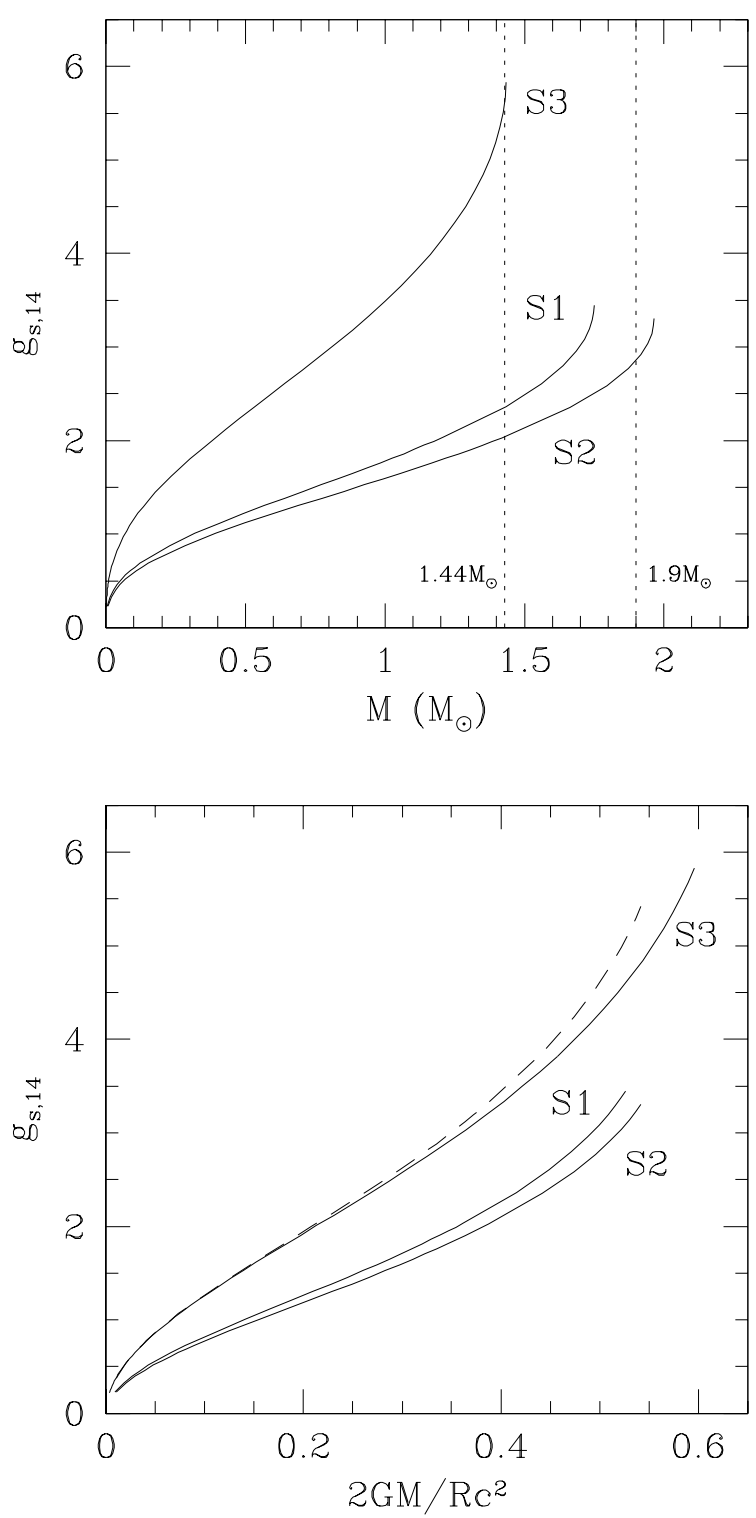

Fig. 4. Surface gravity in units of $10^{14} \mathrm{~cm} \mathrm{~s}^{-2}$ versus strange star mass (upper panel) and compactness parameter (lower panel), for three EOSs of strange quark matter. Dashed line in the lower panel is obtained by transforming (see text for details) the S2 EOS curve into S3 EOS curve using Eq. (7).

simple: the density within such strange stars is nearly constant, so that the dependence on $a$ is negligible.

\section{Discussion and conclusion}

We derived an upper bound on the surface gravity of static neutron stars with subluminal EOSs. Maximum $g_{\mathrm{s}}$ is reached at the maximum allowable mass $M_{\max }$. Even at the shortest observed pulsar period of $1.56 \mathrm{~ms}$ the effect of rotation on the $M_{\max }$ is very small, so that the static approximation is justified. The upper bound on $g_{\mathrm{s}}$ is inversely proportional to the highest measured neutron-star mass $M_{\mathrm{obs}}^{\max }$ and is $7.4 \times 10^{14} \mathrm{~cm} \mathrm{~s}^{-2}$ if $M_{\mathrm{obs}}^{\max }$ is replaced by $1.9 M_{\odot}$, which is the $95 \%$ confidence-level lower bound on mass of the compact star in 4U1700-37 measured by Clark et al. (2002). However, this upper bound on $g_{\mathrm{s}}$ should be taken as highly unreliable. Firstly, Clark et al. (2002) used a specific model of the companion star and of the binary to evaluate the compact-star mass. Secondly, one cannot exclude, unfortunately, that the compact object in 4U1700-37 is actually a low-mass black hole and not a neutron star (Clark et al. 2002 give arguments for and against the black-hole presence).

We have studied $g_{\mathrm{s}}$ for a set of 31 EOSs of baryonic matter. The dependence of $g_{\mathrm{s}}$ on the stellar mass $M$ is very sensitive to the EOS. On the contrary, the dependence of $g_{\mathrm{s}}$ on the stellar compactness $r_{\mathrm{g}} / R$ has a generic character for baryonic EOSs. The maximum surface gravity $g_{\mathrm{s}, \max }$ is sensitive to the EOS of dense matter and ranges from about $2 \times 10^{14} \mathrm{~cm} \mathrm{~s}^{-2}$ to $5 \times$ $10^{14} \mathrm{~cm} \mathrm{~s}^{-2}$ for subluminal baryonic EOSs.

The dependence of $g_{\mathrm{s}}$ on the mass and compactness of strange stars is very different from that of baryonic stars. However, the range of $g_{\mathrm{s}, \max }=(3-6) \times 10^{14} \mathrm{~cm} \mathrm{~s}^{-2}$ is quite similar to baryonic stars.

We hope that the EOS-sensitive features of $g_{\mathrm{s}}$ will be useful in extracting information about the EOS of dense matter, for instance, by combining the values of $g_{\mathrm{s}}$ obtained fitting the thermal component of the observed photon spectra with atmospheric models and the surface redshift measured for the identified spectral lines.

Acknowledgements. This work was motivated by a question of A. Majczyna concerning the possible range of surface gravity in neutron star atmospheres, which is gratefully acknowledged. We are grateful to D. G. Yakovlev for the reading of the manuscript and for helpful remarks. This work was partially supported by the KBN grants No. 2P03D.019.24 and 5P03D.020.20.

\section{References}

Akmal, A., Pandharipande, V. R., \& Ravenhall, D. G. 1998, Phys. Rev. $\mathrm{C}, 58,1804$

Balberg, S., \& Gal, A. 1997, Nucl. Phys. A, 625, 435

Balberg, S., Lichtenstadt, I., \& Cook, G. B. 1999, ApJS, 121, 515

Baldo, M., Bombaci, I., \& Burgio, G. F. 1997, A\&A, 328, 274

Bethe, H. A., \& Johnson, M. B. 1974, Nucl. Phys. A, 230, 1

Bludman, S. A., \& Ruderman, M. A. 1968, Phys. Rev., 170, 1176

Bludman, S. A., \& Ruderman, M. A. 1970, Phys. Rev. D, 1, 3243

Bombaci, I. 1995, in Perspectives on Theoretical Nuclear Physics, ed. I. Bombaci, A. Bonaccorso, A. Fabrocini, et al., p. 223

Clark, J. S., Goodwin, S. P., Crowther, P. A., et al. 2002, A\&A, 392, 909

Dey, M., Bombaci, I., Dey, J., Ray, S., \& Samanta, B. C. 1998, Phys. Lett. B, 438, 123

Douchin, F., \& Haensel, P. 2001, A\&A, 380, 151

Glendenning, N. K. 1985, ApJ, 293, 470

Glendenning, N. K. 1997, Compact Stars: Nuclear Physics, Particle Physics and General Relativity (New York: Springer)

Haensel, P., Kutschera, M., \& Prószyński, M. 1980, A\&A, 102, 299

Haensel, P., Lasota, J. P., \& Zdunik, J. L. 1999, A\&A, 344, 151

Haensel, P., Zdunik, J. L., \& Schaefer, R. 1986, A\&A, 160, 121

Hartle, J. B. 1978, Phys. Repts., 46, 201

Kalogera, V., \& Baym, G. 1996, ApJ, 470, L61

Keiser, B., \& Polyzou, W. 1996, Phys. Rev. C, 54, 2023

Koranda, S., Stergioulas, N., \& Friedman, J. L. 1997, ApJ, 488, 799

Kubis, S. 2001, Ph.D. Thesis, Jagiellonian University (unpublished)

Pandharipande, V. R. 1971, Nucl. Phys. A, 147, 641 
Pandharipande, V. R., \& Ravenhall, D. G. 1989, in Proc. NATO Advanced Research Workshop on nuclear matter and heavy ion collisions, Les Houches 1989, ed. M. Soyeur, et al. (New York: Plenum), 103

Potekhin, A. Y., Yakovlev, D. G., \& Chabrier, G. 2003, ApJ, 594, 404

Rhoades, C. E., \& Ruffini, R. 1974, Phys. Rev. Lett., 32, 324

Shapiro, S. L., \& Teukolsky, S. A. 1983, Black holes, white dwarfs, and neutron stars: The physics of compact objects (New York: Wiley-Interscience)

Muto, T., \& Tatsumi, T. 1990, Prog. Theor. Phys., 83, 499

Walecka, J. D. 1974, Ann. Phys., 83, 491

Weber, F., Glendenning, N. K., \& Weigel, M. K. 1991, ApJ, 373, 579
Weisberg, J. M., \& Taylor, J. H. 2003, in Radio Pulsars, ed. M. Bailes, Nice D. J., \& Thorsett S. E., ASP Conf. Ser., in press [arXiv:astro-ph/0211217]

Wiringa, R. B., Fiks, V., \& Fabrocini, A. 1988, Phys. Rev. C, 38, 1010

Witten, E. 1984, Phys. Rev. D, 30, 272

Zavlin, V. E., \& Pavlov, G. G. 2002, in Proceedings of the 270, WE-Heraeus Seminar on Neutron Stars, Pulsars, and Supernova Remnants, MPE Rep., 278, ed. W. Becker, H. Lesch, \& J. Truemper, Garching bei Muenchen: Max-Planck-Institut fuer extraterrestrische Physik, 263

Zdunik, J. L. 2000, A\&A, 359, 311 